\title{
Kerr-Taub-NUT General Frame, Energy, and Momentum in Teleparallel Equivalent of General Relativity
}

\author{
Gamal G. L. Nashed ${ }^{1,2,3,4}$ \\ ${ }^{1}$ Mathematics Department, Faculty of Science, King Faisal University, P.O. Box 380, \\ Al-Ahsaa 31982, Saudi Arabia \\ ${ }^{2}$ Mathematics Department, Faculty of Science, Ain Shams University, Cairo 11566, Egypt \\ ${ }^{3}$ Center for Theoretical Physics, British University of Egypt, P.O. Box 43, Sherouk City 11837, Egypt \\ ${ }^{4}$ Egyptian Relativity Group (ERG), Egypt
}

Correspondence should be addressed to Gamal G. L. Nashed, nashed@bue.edu.eg

Received 29 December 2011; Revised 16 March 2012; Accepted 1 April 2012

Academic Editor: A. Petrov

Copyright (C) 2012 Gamal G. L. Nashed. This is an open access article distributed under the Creative Commons Attribution License, which permits unrestricted use, distribution, and reproduction in any medium, provided the original work is properly cited.

A new exact solution describing a general stationary and axisymmetric object of the gravitational field in the framework of teleparallel equivalent of general relativity (TEGR) is derived. The solution is characterized by three parameters "the gravitational mass $M$, the rotation $a$, and the NUT L." The vierbein field is axially symmetric, and the associated metric gives the Kerr-TaubNUT spacetime. Calculation of the total energy using two different methods, the gravitational energy momentum and the Riemannian connection 1 -form $\widetilde{\Gamma}_{\alpha}^{\beta}$, is carried out. It is shown that the two methods give the same results of energy and momentum. The value of energy is shown to depend on the mass $M$ and the NUT parameter $L$. If $L$ is vanishing, then the total energy reduced to the energy of Kerr black hole.

\section{Introduction}

One of the generalizations of Kerr solution in general relativity (GR) is done by introducing an extra parameter, the so-called gravitomagnetic monopole moment or magnetic mass. The resulting solution describes the spacetime of a localized stationary and axisymmetric object known as the Kerr-Taub-NUT (Newman-Unti-Tamburino) solution of the vacuum Einstein field equations [1]. This solution belongs to a general class of metrics that admits separability of variables in the Hamilton-Jacobi equations and contains three physical parameters: the gravitational mass $M$ (gravitoelectric charge), the magnetic mass $L$ (NUT charge), and the rotation parameter $a$. The presence of the NUT charge in the spacetime destroys its asymptotic structure making it asymptotically nonflat. 
Attempts at identifying an energy momentum density for gravity has led to various energy momentum complexes which are pseudotensors [2]. Pseudotensors are not covariant objects that is, they essentially depend on the reference frame and thus cannot provide a true physical local gravitational energy momentum density. Hence, the pseudotensor approach has been largely abandoned (cf., $[3,4]$ ).

Teleparallel theories are interesting for several reasons: first of all, GR can be viewed as a particular theory of teleparallelism, and, thus, teleparallelism could be considered, at the very least, as a different point of view that can lead to the same results [5]. Second, in this framework, one can define an energy momentum complex for the gravitational field that is a true tensor under general coordinate transformations, but not under local Lorentz transformation. This is the reason why teleparallelism was reconsidered by Møller when he was studying the problem of defining an energy momentum tensor for the gravitational field $[6,7]$. The idea was taken over by Pellegrini and Plebański that constructed the general Lagrangian for these theories [8]. The third reason why these theories are interesting is that they can be seen as gauge theories of the translation group (not the full Poincaré group), and, thus, they give an alternative interpretation of GR [9-16].

A well-posed and mathematically consistence expression for the gravitational energy has been developed [17]. It arises in the realm of the Hamiltonian formulation of the TEGR [17] and meets several crucial requirements for any acceptable definition of gravitational energy. The gravitational energy momentum $P^{a}$ obtained in the framework of the TEGR has been investigated in the context of several distinct configurations of the gravitational filed. For asymptotically flat spacetimes, $P^{0}$ yields the ADM energy [18].

The objective of the present work is to derive a new exact general axially symmetric solution in TEGR for the gravitational field and then to calculate the total energy using two different methods, the gravitational energy momentum that is coordinate independent and

the other is the Riemannian connection 1-form ${\widetilde{\Gamma_{\alpha}}}^{\beta}$. In Section 2, the derivation of the field equations of the gravitational field is briefly reviewed. A summary of the derivation of energy and angular momentum using the Hamiltonian formulation is presented in Section 2. In Section 3, the general tetrad field with sixteen unknown functions of $r$ and $\phi$ is applied to the field equations. Solving the resulting partial differential equations, a new exact analytic solution is derived, that is, new solution within the context of TEGR. In Section 4, calculation of the energy and spatial momentum using the definitions provided in Section 2 is achieved. In Section 5, the language of forms is employed and a brief review of the field equations and the superpotential used in the calculations of energy and spatial momentum are presented. Using the Riemannian connection 1-form, calculation of energy and momentum is provided. The last section is devoted to discussion.

\section{The TEGR for Gravitation}

In a spacetime with absolute parallelism, the parallel vector field $h_{a}{ }^{\mu}$ identifies the nonsymmetric affine connection

$$
\Gamma_{\mu \nu}^{\mathcal{\lambda}} \stackrel{\text { def. }}{=} h_{a}{ }^{\lambda} h^{a}{ }_{\mu, \nu}
$$

where $h_{a \mu, v}=\partial_{v} h_{a \mu}$ (spacetime indices $\mu, v, \ldots$, and SO $(3,1)$ indices $a, b, \ldots$ run from 0 to 3 . Time and space indices are indicated to $\mu=0, i$, and $a=(0),(i))$. The curvature tensor defined 
by $\Gamma_{\mu \nu}^{\lambda}$ is identically vanishing, however. The metric tensor $g_{\mu v}$ is given by

$$
g_{\mu \nu}=O_{a b} h_{\mu}^{a} h_{\nu}^{b}
$$

with the Minkowski metric $O_{a b}=\operatorname{diag}(+1,-1,-1,-1)$ (Latin indices are raising and lowering with the aid of $O_{a b}$ and $O^{a b}$ ).

Lagrangian density of the gravitational field in the TEGR, in the presence of matter fields, is given by [17] (throughout this paper, we use the relativistic units, $c=G=1$ and $\kappa=8 \pi)$ :

$$
\mathcal{L}_{G}=\sqrt{-g} L_{G}=-\frac{\sqrt{-g}}{16 \pi}\left(\frac{T^{a b c} T_{a b c}}{4}+\frac{T^{a b c} T_{b a c}}{2}-T^{a} T_{a}\right)-L_{m}=-\frac{\sqrt{-g}}{16 \pi} \Sigma^{a b c} T_{a b c}-L_{m},
$$

where $g=\operatorname{det}\left(g_{\mu v}\right)$. The tensor $\Sigma^{a b c}$ is defined by

$$
\Sigma^{a b c} \stackrel{\text { def. }}{=} \frac{1}{4}\left(T^{a b c}+T^{b a c}-T^{c a b}\right)+\frac{1}{2}\left(O^{a c} T^{b}-O^{a b} T^{c}\right) .
$$

$T^{a b c}$ and $T^{a}$ are the torsion tensor and the basic vector field defined by

$$
\begin{gathered}
T_{\mu \nu}^{a} \stackrel{\text { def. }}{=} h_{\lambda}^{a} T_{\mu \nu}^{\lambda}=\partial_{\mu} h^{a}{ }_{\nu}-\partial_{\nu} h_{\mu}^{a} \\
T^{\mu} \stackrel{\text { def. }}{=} T_{\nu}^{v}{ }_{\nu}{ }^{\mu}, \quad T^{a} \stackrel{\text { def. }}{=} h_{\mu}^{a} T^{\mu}=T_{b}^{b}{ }_{b}^{a} .
\end{gathered}
$$

The quadratic combination $\Sigma^{a b c} T_{a b c}$ is proportional to the scalar curvature $R$, except for a total divergence term [19]. $L_{m}$ represents the Lagrangian density for matter fields.

The gravitational field equations for the system described by $L_{G}$ are the following:

$$
h_{a \lambda} h_{b \mu} \partial_{v}\left(\sqrt{-g} \Sigma^{b \lambda v}\right)-\sqrt{-g}\left(\Sigma^{b v} T_{b v \mu}-\frac{1}{4} h_{a \mu} T_{b c d} \Sigma^{b c d}\right)=\frac{1}{2} \kappa \sqrt{-g} T_{a \mu}
$$

where

$$
\frac{\delta L_{m}}{\delta h^{a \mu}} \equiv \sqrt{-g} T_{a \mu}
$$

It is possible to prove by explicit calculations that the left-hand side of the symmetric part of the field equations (2.7) is exactly given by [17]

$$
\frac{\sqrt{-g}}{2}\left[R_{a \mu}-\frac{1}{2} h_{a \mu} R\right] \text {. }
$$

The axial-vector part of the torsion tensor $A_{\mu}$ is defined by

$$
A_{\mu} \stackrel{\text { def. }}{=} \frac{1}{6} \epsilon_{\mu \nu \rho \sigma} T^{\nu \rho \sigma}=\frac{1}{3} \epsilon_{\mu \nu \rho \sigma} \gamma^{\nu \rho \sigma}, \quad \text { where } \epsilon_{\mu \nu \rho \sigma} \stackrel{\text { def. }}{=} \sqrt{-g} \delta_{\mu \nu \rho \sigma},
$$


with $\gamma_{v \rho \sigma}=O^{a b} h_{a v} h_{b \rho ; \sigma}$ being the contorsion tensor and $\delta_{\mu \nu \rho \sigma}$ is completely antisymmetric and normalized as $\delta_{0123}=-1$.

The definition of the gravitational energy momentum $P^{a}$ four-vector has the form [17]

$$
P^{a}=-\int_{V} d^{3} x \partial_{i} \Pi^{a i}
$$

where $V$ is an arbitrary volume of the three-dimensional space. In the configuration space, we have

$$
\begin{array}{r}
\Pi^{a i}=-\frac{2}{\mathcal{\kappa}} \sqrt{-g} \Sigma^{a 0 i}, \quad \text { with } \partial_{\nu}\left(\sqrt{-g} \Sigma^{a \lambda \nu}\right)=\frac{\kappa}{2} \sqrt{-g} h^{a}{ }_{\mu}\left(t^{\lambda \mu}+T^{\lambda \mu}\right), \\
\text { where } t^{\lambda \mu}=\frac{1}{2 \mathcal{\kappa}}\left(4 \Sigma^{b c \lambda} T_{b c}{ }^{\mu}-g^{\lambda \mu} \Sigma^{b c d} T_{b c d}\right) .
\end{array}
$$

Maluf and Ulhoa [20] defined

$$
L^{a b}=2 \int_{V} d^{3} x M^{[a b]}, \quad \text { where } M^{a b}=\frac{\sqrt{-g}}{4 \pi}\left(\Sigma^{b 0 a}-\Sigma^{a 0 b}\right),
$$

as the 4-angular momentum of the gravitational field for an arbitrary volume $V$ of the threedimensional space.

\section{Exact Analytic Solution}

Let us begin with the general tetrad field which can be written in the polar coordinates as

$$
\left(h^{i}{ }_{\mu}\right)=\left(\begin{array}{cccc}
A_{1}(r, \phi) & A_{2}(r, \phi) & A_{3}(r, \phi) & A_{4}(r, \phi) \\
B_{1}(r, \phi) \sin \theta \cos \phi & B_{2}(r, \phi) \sin \theta \cos \phi & B_{3}(r, \phi) \cos \theta \cos \phi & B_{4}(r, \phi) \sin \phi \sin \theta \\
C_{1}(r, \phi) \sin \theta \sin \phi & C_{2}(r, \phi) \sin \theta \sin \phi & C_{3}(r, \phi) \cos \theta \sin \phi & C_{4}(r, \phi) \cos \phi \sin \theta \\
D_{1}(r, \phi) \cos \theta & D_{2}(r, \phi) \cos \theta & D_{3}(r, \phi) \sin \theta & D_{4}(r, \phi) \cos \theta
\end{array}\right),
$$

where $A_{i}(r, \phi), B_{i}(r, \phi), C_{i}(r, \phi)$, and $D_{i}(r, \phi), i=1, \ldots, 4$, are unknown functions of $r$ and $\phi$. Applying (3.1) to the field equations (2.7), we get a set of nonlinear partial differential equations. (These calculations have been checked using Maple software 13.) Due to the lengthy of writing the output partial differential equations, we will write only the solution that satisfies such differential equations. Here, we list the boundary conditions used to derive the solution. The parallel vector field should have the expression

$$
h^{k}{ }_{\mu}=\delta_{\mu}^{k}+M l^{k} l_{\mu}-\frac{L^{2}}{2} m^{k} m_{\mu}
$$

where $M$ and $L$ are free parameters, $l_{\mu}$ and $m_{\mu}$ are quantities satisfying the conditions

$$
O^{\mu v} l_{\mu} l_{v}=0, \quad O^{\mu v} m_{\mu} m_{v}=0, \quad O^{\mu v} l_{\mu} m_{v}=0,
$$


$l^{k}$ and $m^{k}$ are defined by

$$
l^{k}=\delta^{k}{ }_{\mu} O^{\mu v} l_{v} \quad m^{k}=\delta^{k}{ }_{\mu} O^{\mu v} m_{v}
$$

The solution satisfying the above condition is found to have the following form.

\section{The Exact Solution}

If the arbitrary functions take the following values:

$$
\begin{aligned}
A_{1} & =1-\frac{\left(M r+L L_{1}\right)}{\Omega}, \quad A_{2}=\frac{\left(M r+L L_{1}\right)}{\Upsilon}, \quad A_{3}=0, \\
A_{4} & =\frac{M r \chi+L\left(a L_{1}\left[1+\cos ^{2} \theta\right]+2 r^{2} \cos \theta\right)}{\Omega}, \quad B_{1}=\frac{\left(M r+L L_{1}\right)}{\Omega}, \\
B_{2} & =\frac{1}{\Upsilon \cos \phi}\left(r_{1} \alpha-\left(M r+L L_{1}\right) \cos \phi\right), \quad B_{3}=\frac{\alpha}{\cos \phi^{\prime}}, \quad B_{4}=\frac{\beta-\left(\left(M r+L L_{1}\right) \chi \cos \phi / \Omega\right)}{\sin \phi}, \\
C_{1} & =\frac{\left(M r+L L_{1}\right)}{\Omega}, \quad C_{2}=\frac{1}{\Upsilon \sin \phi}\left(r_{1} \beta-\left(M r+L L_{1}\right) \sin \phi\right), \quad C_{3}=\frac{\beta}{\sin \phi^{\prime}}, \quad D_{2}=1+\frac{\left(M r+L L_{1}\right)}{\Upsilon}, \\
C_{4} & =\frac{-\alpha+\left(M r+L L_{1}\right) \chi \sin \phi / \Omega}{\cos \phi}, \quad D_{1}=\frac{\left(M r+L L_{1}\right)}{\Omega}, \\
D_{3} & =-r_{1}, \quad D_{4}=\frac{-\left(M r+L L_{1}\right) \chi}{\Omega},
\end{aligned}
$$

where $\Omega, \Upsilon, L_{1}, \alpha, \beta, r_{1}$, and $\chi$ are defined by

$$
\begin{aligned}
& \Omega \stackrel{\text { def. }}{=} r^{2}+L_{1}{ }^{2}, \quad \Upsilon \stackrel{\text { def. }}{=} r^{2}+a^{2}-2 M r-L^{2}, \quad L_{1} \stackrel{\text { def. }}{=} L+a \cos \theta, \\
& \alpha \stackrel{\text { def. }}{=} r_{1} \cos \phi+a \sin \phi, \quad \beta \stackrel{\text { def. }}{=} r_{1} \sin \phi-a \cos \phi, \quad r_{1} \stackrel{\text { def. }}{=} \sqrt{r^{2}+L(L+2 a \cos \theta)} \text {, } \\
& x \stackrel{\text { def. }}{=} a \sin ^{2} \theta-2 L \cos \theta \text {. }
\end{aligned}
$$

$M, a$, and $L$ are the gravitational mass, the angular momentum of the rotating source, and the NUT parameter $[1,21]$.

The parallel vector field (3.1), using solution (3.5), is axially symmetric in the sense that it is form invariant under the transformations

$$
\begin{aligned}
& \bar{\phi} \longrightarrow \phi+\delta \phi, \quad \overline{h^{(0)}}{ }_{\mu} \longrightarrow h^{(0)}{ }_{\mu}, \quad \overline{h^{(1)}}{ }_{\mu} \longrightarrow h^{(1)}{ }_{\mu} \cos \delta \phi-h^{(2)}{ }_{\mu} \sin \delta \phi \text {, } \\
& \overline{h^{(2)}}{ }_{\mu} \longrightarrow h^{(2)}{ }_{\mu} \sin \delta \phi+h^{(2)}{ }_{\mu} \cos \delta \phi, \quad \overline{h^{(3)}}{ }_{\mu} \longrightarrow h^{(3)}{ }_{\mu} \text {. }
\end{aligned}
$$


Solution (3.5) satisfies the field equations (2.7), and the associated metric has the following form:

$$
\begin{aligned}
d s^{2}= & \frac{1}{\Omega}\left\{\Upsilon-a^{2} \sin ^{2} \theta\right\} d t^{2}-\frac{\Omega}{\Upsilon} d r^{2}-\Omega d \theta^{2}-\frac{1}{\Omega}\left\{\left(\Omega+a_{X}\right)^{2} \sin ^{2} \theta-\Upsilon X^{2}\right\} d \phi^{2} \\
& -\frac{2}{\Omega}\left\{X \Upsilon-a\left(\Omega+a_{X}\right) \sin ^{2} \theta\right\} d t d \phi,
\end{aligned}
$$

which is the Kerr-Taub-NUT black hole in the Boyer-Lindquist coordinate [22, 23].

The earlier obtained solutions Schwarzschild spacetime could be generated as special solutions of the tetrad (3.1) using (3.5) by putting $L=0, a=0$, and kerr spacetime can be obtained when $L=0[21,23]$.

\section{Energy Associated with the Axially Symmetric Solution}

Now, we are going to calculate the energy content of the tetrad field (3.1) using (3.5). The nonvanishing components of the tensor $\Sigma^{a b c}$ needed to the calculation of energy have the form

$$
\begin{aligned}
& \Sigma^{101}=\frac{a}{2 r^{5}}\left(\left\{a L L_{1}+a M r\right\} \sin ^{2} \theta-4 a L^{2} \cos ^{2} \theta-2 M r L \cos \theta\right), \\
& \Sigma^{201}=\frac{a}{2 r^{5} \sin \theta}\left(L r \sin ^{2} \theta+2 L M \cos ^{2} \theta-M a \cos \theta \sin ^{2} \theta\right), \\
& \Sigma^{301}=\frac{L}{2 r^{5} \sin ^{2} \theta}\left(L^{2} \cos \theta-a L_{1} \sin ^{2} \theta\right), \\
& \Sigma^{401}=-\frac{1}{4 r^{5}}\left(a^{2}\left\{5 L^{2}+2 M r\right\} \sin ^{2} \theta+4 a^{2}\left(r^{2}-9 L^{2}\right) \cos ^{2} \theta+4 L r\left[\{r-M\} a \cos \theta+r L_{1}\right]-4 r^{4}\right) .
\end{aligned}
$$

Using (3.6) in (2.11), we finally obtain (we subtract $\Sigma_{M=0, a=0, L=0}^{\mu 0 k}$ in (4.2) to remove divergences from the calculations of energy)

$$
\begin{aligned}
P^{(0)} & =E=-\oint_{S \rightarrow \infty} d S_{k} \Pi^{(0) k}=-\frac{1}{4 \pi} \oint_{S \rightarrow \infty} d S_{k} \sqrt{-g} h_{\mu}^{(0)}\left(\Sigma^{\mu 0 k}-\Sigma_{M=0, a=0, L=0}^{\mu 0 k}\right) \\
& \cong M+\frac{L^{2}}{r}-\frac{L^{2} M}{r^{2}}-\frac{L^{2}\left(5 a^{2}+L^{2}\right)}{r^{3}}+O\left(\frac{1}{r^{4}}\right) .
\end{aligned}
$$

The total energy, when $r \rightarrow \infty$, gives the ADM (Arnowitt-Deser-Misner) mass.

The nonvanishing components needed to calculate the spatial momentum have the form (terms like $M^{2}, L^{3}, L^{3} M, M^{2} a, \ldots$, etc. are neglected in this calculations)

$$
\begin{aligned}
& \Sigma^{(1) 01} \cong \frac{\sin ^{2} \theta \cos \phi}{4 \pi r}\left(M r+L^{2}\right)+O\left(\frac{1}{r^{2}}\right), \quad \Sigma^{(2) 01} \cong \frac{-\sin ^{2} \theta \sin \phi}{4 \pi r}\left(M r+L^{2}\right)+O\left(\frac{1}{r^{2}}\right), \\
& \Sigma^{(3) 01} \cong \frac{-\sin ^{2} \theta \cos \phi}{4 \pi r}\left(M r+L^{2}\right)+O\left(\frac{1}{r^{2}}\right) .
\end{aligned}
$$


Using (4.3) in (2.11), we finally get the spatial momentum in the form

$$
\begin{gathered}
P_{1}=-\oint_{S \rightarrow \infty} d S_{k} \Pi^{(1) k}=-\frac{1}{4 \pi} \oint_{S \rightarrow \infty} d S_{k} e \Sigma^{(1) 0 k}=0, \\
\quad \text { by same method } P_{2}=0, \quad P_{3} \cong\left(\frac{1}{r}\right) .
\end{gathered}
$$

The nonvanishing components of the angular-momentum are given by

$$
\begin{aligned}
& M^{(0)(1)}(r, \theta, \phi) \cong \frac{1}{8 \pi r}\left(\left\{6 L a \cos \theta+2 r M+a^{2}+3 L^{2}\right\} \cos ^{2} \theta \cos \phi+r a\left\{\cos ^{2} \theta+1\right\} \sin \phi\right. \\
& \left.+\left\{2 r^{2}-2 L^{2}-2 M r-a^{2}-4 L a \cos \theta\right\} \cos \phi\right)+O\left(\frac{1}{r^{2}}\right), \\
& M^{(0)(2)}(r, \theta, \phi) \cong \frac{1}{8 \pi r}\left(\left\{6 L a \cos \theta+2 r M+a^{2}+3 L^{2}\right\} \cos ^{2} \theta \sin \phi-r a\left\{\cos ^{2} \theta+1\right\} \cos \phi\right. \\
& \left.+\left\{2 r^{2}-2 L^{2}-2 M r-a^{2}-4 L a \cos \theta\right\} \sin \phi\right)+O\left(\frac{1}{r^{2}}\right), \\
& M^{(0)(3)}(r, \theta, \phi) \cong \frac{\sin \theta\left(6 L a \cos ^{2} \theta+\left[2 r M+3 L^{2}\right] \cos \theta-2 L h\right)}{8 \pi r}+O\left(\frac{1}{r^{2}}\right) \text {, } \\
& M^{(1)(2)}(r, \theta, \phi) \cong \frac{\sin \theta\left(3 M a \cos ^{2} \theta+2[M-r] L \cos \theta-M a\right)}{8 \pi r}+O\left(\frac{1}{r^{2}}\right), \\
& M^{(1)(3)}(r, \theta, \phi) \cong \frac{\sin ^{2} \theta([3 M a \cos \theta+2 L M-2 L r] \sin \phi+L a \cos \phi)}{8 \pi r}+O\left(\frac{1}{r^{2}}\right), \\
& M^{(2)(3)}(r, \theta, \phi) \cong \frac{\sin ^{2} \theta([3 M a \cos \theta+2 L M-2 L r] \cos \phi-L a \sin \phi)}{8 \pi r}+O\left(\frac{1}{r^{2}}\right) .
\end{aligned}
$$

Using (4.5) in (2.13), we get

$$
L^{(0)(1)}=\int_{0}^{\pi} \int_{0}^{2 \pi} \int_{0}^{\infty} d \theta d \phi d r\left[M^{(0)(1)}\right]=0,
$$

which is a consistent result. By the same method, we finally obtain

$$
L^{(0)(2)}=L^{(0)(3)}=L^{(1)(2)}=L^{(1)(3)}=L^{(2)(3)}=0 .
$$

We show by explicit calculation that the energy momentum tensor which is a coordinate independent does not give a consistent result of the angular momentum when applied to the tetrad field given by (3.1)! The same problem appeared in ([24], equation (44)) when calculation of angular momentum of the BTZ black hole has been implemented. We may claim that the unusual result of the angular momentum is related to the use of expression (2.13). May be possible solution to this problem is to use other definition or to calculate the total conserved quantities of solution (3.5). This will be our forthcoming research work. 


\section{Brief Review of Teleparallel Gravity Using the Language of Forms}

\subsection{Notation}

We use the Latin indices $i, j, \ldots$, for local holonomic spacetime coordinates and the Greek indices $\alpha, \beta, \ldots$, label (co)frame components. Particular frame components are denoted by hats, $\widehat{0}, \widehat{1}$, and so forth. As usual, the exterior product is denoted by $\wedge$, while the interior product of a vector $\xi$ and a $p$-form $\Psi$ is denoted by $\xi \mid \Psi$. The vector basis dual to the frame 1 -forms $\vartheta^{\alpha}$ is denoted by $e_{\alpha}$, and they satisfy $e_{\alpha} \mid \vartheta^{\beta}=\delta_{\alpha}{ }^{\beta}$. Using local coordinates $x^{i}$, we have $\vartheta^{\alpha}=h_{i}^{\alpha} d x^{i}$ and $e_{\alpha}=h_{\alpha}^{i} \partial_{i}$ where $h_{i}^{\alpha}$ and $h_{\alpha}^{i}$ are the covariant and contravariant components of the tetrad field. We define the volume 4-form by $\eta \stackrel{\text { def. }}{=} \vartheta^{\hat{0}} \wedge \vartheta^{\hat{1}} \wedge \vartheta^{\hat{2}} \wedge \vartheta^{\hat{3}}$. Furthermore, with the help of the interior product, we define

$$
\left.\eta_{\alpha} \stackrel{\text { def. }}{=} e_{\alpha}\right\rfloor \eta=\frac{1}{3 !} \epsilon_{\alpha \beta \gamma \delta} \vartheta^{\beta} \wedge \vartheta^{r} \wedge \vartheta^{\delta},
$$

where $\epsilon_{\alpha \beta \gamma \delta}$ is completely antisymmetric tensor with $\epsilon_{0123}=1$

$$
\left.\left.\eta_{\alpha \beta} \stackrel{\text { def. }}{=} e_{\beta}\right\rfloor \eta_{\alpha}=\frac{1}{2 !} \epsilon_{\alpha \beta \gamma \delta} \vartheta^{\gamma} \wedge \vartheta^{\delta}, \quad \eta_{\alpha \beta \gamma} \stackrel{\text { def. }}{=} e_{\gamma}\right\rfloor \eta_{\alpha \beta}=\frac{1}{1 !} \epsilon_{\alpha \beta \gamma \delta} \vartheta^{\delta},
$$

which are bases for 3-, 2-, and 1-forms, respectively. Finally,

$$
\left.\left.\left.\left.\left.\eta_{\alpha \beta \mu \nu} \stackrel{\text { def. }}{=} e_{\nu}\right\rfloor \eta_{\alpha \beta \mu}=e_{v}\right\rfloor e_{\mu}\right\rfloor e_{\beta}\right\rfloor e_{\alpha}\right\rfloor \eta
$$

is the Levi-Civita tensor density. The $\eta$-forms satisfy the useful identities:

$$
\begin{aligned}
\vartheta^{\beta} \wedge \eta_{\alpha} \stackrel{\text { def. }}{=} \delta_{\alpha}^{\beta} \eta, \quad \vartheta^{\beta} \wedge \eta_{\mu \nu} \stackrel{\text { def. }}{=} \delta_{v}^{\beta} \eta_{\mu}-\delta_{\mu}^{\beta} \eta_{v}, \quad \vartheta^{\beta} \wedge \eta_{\alpha \mu \nu} \stackrel{\text { def. }}{=} \delta_{\alpha}^{\beta} \eta_{\mu \nu}+\delta_{\mu}^{\beta} \eta_{v \alpha}+\delta_{v}^{\beta} \eta_{\alpha \mu \nu} \\
v^{\beta} \wedge \eta_{\alpha \gamma \mu \nu} \stackrel{\text { def. }}{=} \delta_{v}^{\beta} \eta_{\alpha \gamma \mu}-\delta_{\mu}^{\beta} \eta_{\alpha \gamma v}+\delta_{\gamma}^{\beta} \eta_{\alpha \mu \nu}-\delta_{\alpha}^{\beta} \eta_{\gamma \mu \nu} .
\end{aligned}
$$

The line element $d s^{2} \stackrel{\text { def. }}{=} g_{\alpha \beta} \vartheta^{\alpha} \otimes \vartheta^{\beta}$ is defined by the spacetime metric $g_{\alpha \beta}$.

Teleparallel geometry can be viewed as a gauge theory of translation [10-16, 2528]. In this geometry, the coframe $\vartheta^{\alpha}$ plays the role of the gauge translational potential of the gravitational field. GR can be reformulated as the teleparallel theory. Geometrically, teleparallel gravity can be considered as a special case of the metric-affine gravity in which the coframe 1-form $\vartheta^{\alpha}$ and the local Lorentz connection are subject to the distant parallelism constraint $R_{\alpha}^{\beta}=0$ [29-38]. In this geometry, the torsion 2-form

$$
T^{\alpha}=D \vartheta^{\alpha}=d \vartheta^{\alpha}+\Gamma_{\beta}{ }^{\alpha} \wedge \vartheta^{\beta}=\frac{1}{2} T_{\mu \nu}{ }^{\alpha} \vartheta^{\mu} \wedge \vartheta^{\nu}=\frac{1}{2} T_{i j}{ }^{\alpha} d x^{i} \wedge d x^{j}
$$

arises as the gravitational gauge field strength, $\Gamma_{\alpha}{ }^{\beta}$ being the Weitzenböck connection 1 -form, $d$ is the total derivative, and $D$ is the covariant derivative. The torsion $T^{\alpha}$ can be decomposed 
into three irreducible pieces: the tensor part, the trace, and the axial trace, given, respectively, by $[9,11-16]$, for example,

${ }^{(1)} T^{\alpha} \stackrel{\text { def. }}{=} T^{\alpha}-{ }^{(2)} T^{\alpha}-{ }^{(3)} T^{\alpha}$, with

${ }^{(2)} T^{\alpha} \stackrel{\text { def. }}{=} \frac{1}{3} \vartheta^{\alpha} \wedge T$, where $\left.\left.T=\left(e_{\beta}\right\rfloor T^{\beta}\right), e_{\alpha}\right\rfloor T=T_{\mu \alpha}{ }^{\mu}$, vectors of trace of torsion,

$\left.{ }^{(3)} T^{\alpha} \stackrel{\text { def. }}{=} \frac{1}{3} e^{\alpha}\right\rfloor P, \quad$ with $\left.P=\left(\vartheta^{\beta} \wedge T_{\beta}\right), e_{\alpha}\right\rfloor P=T^{\mu \nu \lambda} \eta_{\mu \nu \lambda \alpha}$, axial of trace of torsion.

The Lagrangian of the teleparallel equivalent using the language of forms has the form

$$
V=-\frac{1}{2 \kappa} T^{\alpha} \wedge^{*}\left({ }^{(1)} T_{\alpha}-2^{(2)} T_{\alpha}-\frac{1}{2}{ }^{(3)} T_{\alpha}\right)
$$

$\kappa=8 \pi G / c^{3}, G$ is the Newtonian constant, $c$ is the speed of light, and $*$ denotes the Hodge duality in the metric $g_{\alpha \beta}$ which is assumed to be flat Minkowski metric $g_{\alpha \beta}=O_{\alpha \beta}=$ $\operatorname{diag}(+1,-1,-1,-1)$, that is used to raise and lower local frame (Greek) indices.

The variation of the total action with respect to the coframe gives the field equations in the from [9]

$$
D H_{\alpha}-E_{\alpha}=\Sigma_{\alpha}, \quad \text { where } \Sigma_{\alpha} \stackrel{\text { def. }}{=} \frac{\delta L_{\text {mattter }}}{\delta \vartheta^{\alpha}}
$$

is the canonical energy momentum current 3-form of matter which is considered as the source. In accordance with the general Lagrange-Noether scheme [10, 25], one derives from (5.7) the translational momentum 2-form and the canonical energy momentum 3-form:

$$
\left.\left.H_{\alpha} \stackrel{\text { def. }}{=}-\frac{\partial V}{\partial T^{\alpha}}=\frac{1}{\kappa} *\left({ }^{(1)} T_{\alpha}-2^{(2)} T_{\alpha}-\frac{1}{2}{ }^{(3)} T_{\alpha}\right), \quad E_{\alpha} \stackrel{\text { def. }}{=} \frac{\partial V}{\partial \vartheta^{\alpha}}=e_{\alpha}\right\rfloor V+\left(e_{\alpha}\right\rfloor T^{\beta}\right) \wedge H_{\beta} .
$$

Due to geometric identities [39], the Lagrangian (5.7) can be recast as

$$
V=-\frac{1}{2} T^{\alpha} \wedge H_{\alpha}
$$

The presence of the connection field $\Gamma_{\beta}^{\alpha}$ plays an important regularizing role due to the following [40].

First

The theory becomes explicitly covariant under the local Lorentz transformations of the coframe, that is, the Lagrangian (5.7) is invariant under the change of variables

$$
\vartheta^{\prime \alpha}=\Lambda_{\beta}^{\alpha} \vartheta^{\beta}, \quad \Gamma_{\alpha}^{\prime \beta}=(\Lambda)_{\alpha}^{\mu} \Gamma_{\mu}^{v}\left(\Lambda^{-1}\right)_{v}^{\beta}-\left(\Lambda^{-1}\right)_{\gamma}^{\beta} d(\Lambda)_{\alpha}^{\gamma} .
$$


Due to the noncovariant transformation law of $\Gamma_{\alpha}{ }^{\beta}$ as shown by (5.11), if a connection vanishes in a given frame, it will not vanish in any other frame related to the first by a local Lorentz transformation.

\section{Second}

$\Gamma_{\alpha}{ }^{\beta}$ plays an important role in the teleparallel framework. This role represents the inertial effects which arise from the choice of the reference system [9]. The contributions of this inertial in many cases lead to unphysical results for the total energy of the system. Therefore, the role of the teleparallel connection is to separate the inertial contribution from the truly gravitational one. Since the teleparallel curvature is zero, the connection is pure gauge, that is

$$
\Gamma_{\alpha}^{\beta}=\left(\Lambda^{-1}\right)_{\gamma}^{\beta} d(\Lambda)_{\alpha}^{\gamma}
$$

The Weitzenböck connection always has the form (5.12). The translational momentum has the form [9]

$$
\widetilde{H}_{\alpha}=\frac{1}{2 \mathcal{K}} \widetilde{\Gamma}^{\beta \gamma} \wedge \eta_{\alpha \beta \gamma}, \quad \Gamma_{\alpha} \stackrel{\text { def. }}{=}{\widetilde{\Gamma_{\alpha}}}^{\beta}-K_{\alpha}^{\beta},
$$

with $\widetilde{\Gamma_{\alpha}}{ }^{\beta}$ is the purely Riemannian connection and $K^{\mu v}$ is the contorsion 1-form which is related to the torsion through the relation

$$
T^{\alpha} \stackrel{\text { def. }}{=} K_{\beta}^{\alpha} \wedge \vartheta^{\beta} \text {. }
$$

Using the spherical local coordinates $(t, r, \theta, \phi)$, the Kerr-Taub-NUT, using (3.1), frame is described by the coframe components:

$$
\begin{aligned}
& \vartheta^{\delta}=(h)_{i}^{\delta} d x^{i}, \\
& \vartheta^{\hat{0}}=A_{1} c d t+A_{2} d r+A_{3} d \theta+A_{4} d \phi, \\
& \vartheta^{\hat{1}}=B_{1} \sin \theta \cos \phi c d t+B_{2} \sin \theta \cos \phi d r+B_{3} \cos \theta \cos \phi d \theta+B_{4} \sin \theta \sin \phi d \phi, \\
& \vartheta^{\hat{2}}=C_{1} \sin \theta \sin \phi c d t+C_{2} \sin \theta \sin \phi d r+C_{3} \cos \theta \sin \phi d \theta+C_{4} \sin \theta \cos \phi d \phi, \\
& \vartheta^{\widehat{3}}=D_{1} \cos \theta c d t+D_{2} \cos \theta d r+D_{3} \sin \theta d \theta+D_{4} \cos \theta d \phi .
\end{aligned}
$$

If we take coframe (5.15), as well as the Riemannian connection ${\widetilde{\Gamma_{\alpha}}}^{\beta}$ and substitute into (5.13), we finally get ( $\cdots$ means terms which are multiply by $d \theta \wedge d r, d \theta \wedge d t, d r \wedge d \phi$, etc.).

$$
\widetilde{H}_{\widehat{0}} \cong \frac{\sin \theta}{4 r \pi}\left[\left(M r+L L_{1}\right)\right](d \theta \wedge d \phi)+\cdots+O\left(\frac{1}{r^{2}}\right) .
$$


Using (5.16) to compute the total energy at a fixed time in the 3-space with a spatial boundary 2-dimensional surface $\partial S=\{r=R, \theta, \phi\}$, we obtain

$$
\widetilde{E}=\int_{\partial S} \widetilde{H}_{\hat{0}}=\left\{M+\frac{L^{2}}{R}\right\}+O\left(\frac{1}{R^{2}}\right),
$$

which is identical with (4.2).

Turn our attention to the spatial momentum, we get the following nonvanishing components $\widetilde{H}_{\widehat{\alpha}}, \widehat{\alpha}=1,2,3$,

$$
\begin{aligned}
& \widetilde{H}_{\hat{1}}=\frac{\cos \theta\left(M r+L L_{1}\right)}{4 r \pi}(d \theta \wedge d \phi)+\cdots+O\left(\frac{1}{r^{2}}\right), \\
& \widetilde{H}_{\hat{2}}=\frac{2 L a \sin ^{2} \theta}{8 r \pi}(d \theta \wedge d \phi)+\cdots+O\left(\frac{1}{r^{2}}\right), \\
& \widetilde{H}_{\widehat{3}}=\frac{2 L \cos \theta(r-M)+M a \sin ^{2} \theta}{8 r \pi}(d \theta \wedge d \phi)+\cdots+O\left(\frac{1}{r^{2}}\right) .
\end{aligned}
$$

Using (5.18) in (5.13), we finally get the spatial momentum in the form

$$
P_{1}=\int_{\partial S} \widetilde{H}_{\hat{1}}=O\left(\frac{1}{R}\right), \quad \text { by same method } P_{2}=P_{3}=O\left(\frac{1}{R}\right) .
$$

\section{Main Results and Discussion}

In this paper, the equations of the gravitational field in the TEGR are considered, applying the most general tetrad (3.1) with sixteen unknown function of $r$ and $\phi$ to the field equations (2.7). Exact analytic new solution is obtained satisfying (3.2). This solution is a general one from which other solutions can be generated (Schwarzschild and Kerr spacetimes) by appropriate choice of the arbitrary functions of the tetrad equation (3.1) using (3.5), that is, putting $L=0, a=0$, and kerr spacetime can be obtained when $L=0[21,23]$. The tetrad field is axially symmetric as shown in (3.7).

By using the definition of the torsion tensor provided by (2.5) and appling it to the tetrad field (3.1), using (3.5), one can show that the torsion of flat spacetime is vanishing identically. Therefore, the gravitational energy momentum given by (2.11) is employed. Use of (2.11) is not limited to Cartesian coordinate. Accordingly, (2.11) is applied to the tetrad field (3.1), using (3.5), and the energy content (4.2) is derived. Equation (4.2) indicates clearly that the energy content is shared by the gravitational mass $M$ and the NUT parameter $L$ of the Kerr-Taub-NUT black hole. This result is agreed with what has been obtained before $[22,23]$. Setting the NUT parameter $L$ equal zero, expression (4.2) gives the energy of the Kerr black hole [23]. With the assistance of gravitational energy momentum, calculation of the spatial momentum has been accomplished and results are consistent [18]. Calculations of the angular momentum components are achieved using definition (2.13). The calculations using this definition are not agreed with the previous one, since all the components are vanishing [41]!

To use another definition to calculate energy and angular momentum of tetrad (3.1), the language of forms is employed and the Riemannian connection, that is, $\widetilde{\Gamma}_{\alpha}^{\beta}$, is presented. 
The formulas of energies as shown by (4.2) and (5.17) indicate that the two methods, the energy momentum tensor and the translational momentum 2-form $H_{\alpha}$, gave the same value of energy and spatial momentum. Consistence of the results of two methods is due to the following.

(i) Within the context of tetrad theories of gravity, asymptotically flat spacetimes may be featured by the asymptotic boundary condition

$$
e_{a \mu} \cong \eta_{a \mu}+\frac{1}{2} h_{a \mu}\left(\frac{1}{r}\right)
$$

and by the condition $\partial_{\mu} e^{a}{ }_{\mu}=O\left(1 / r^{2}\right)$ in the asymptotic limit $r \rightarrow \infty$. It is obvious that the tetrad field (3.1) using (3.5) satisfied this condition.

(ii) Components of torsion related to solution (3.5) are vanishing when the physical quantities are set equal to zero, that is, $M, a, L$. This is a prerequisite to get eligible results [17].

(iii) When the coframe $\vartheta^{\alpha}$, taken as the only field variable in the formulation of purely tetrad and in order to obtain physical results, one should select a tetrad field that meets certain conditions.

(a) The asymptotic behavior of Riemannian connection ${\widetilde{\Gamma_{\beta}}}^{\alpha}$ must behave like $O(1 / r)[9]$.

(b) When the physical quantities, that is, $M, a, L$, are set equal to zero then Riemannian connection is vanishing, that is, $\left(\widetilde{\Gamma_{\beta}^{\alpha}}\right)_{M, a, L=0} \rightarrow 0$.

(iv) Definition (2.13), related to the calculation of angular momentum, does not seem working properly for the Kerr-NUT spacetime. This problem needs an extensive study, and this will be our future work.

\section{Acknowledgment}

The author acknowledges the Egyptian Relativity Group (http:/ /www.erg.eg.net/), in which he is a member.

\section{References}

[1] M. Demiański and E. T. Newman, “Algebraically special of Petrov type II with repeated principal null vector P," Bulletin de l'Académie Polonaise des Sciences, vol. 14, p. 653, 1966.

[2] L. D. Landau and E. M. Lifshitz, The Classical Theory of Fields, Pergamon Press, Oxford, UK, 1980.

[3] M. Dubois-Violette and J. Madore, "Conservation laws and integrability conditions for gravitational and Yang-Mills field equations," Communications in Mathematical Physics, vol. 108, no. 2, pp. 213-223, 1987.

[4] J. Frauendiener, "Geometric description of energy-momentum pseudotensors," Classical and Quantum Gravity, vol. 6, no. 12, pp. L237-L241, 1989.

[5] T. Ortín, Gravity and Strings, Cambridge University Press, Cambridge, UK, 2004.

[6] C. Møller, "Tetrad fields and conservation laws in general relativity," in Proceedings of the International School of Physics Enrico Fermi, C. Mlle, Ed., Academic Press, London, UK, 1962. 
[7] C. Møller, "Conservation laws in the tetrad theory of gravitation," in Proceedings of the Conference on Theory of Gravitation, Warszawa and Jablonna, 1962, vol. 136 of NORDITA Publications, Gauthier-Villars, Paris, France, PWN-Polish Scientific Publishers, Warsaw, Poland, 1964.

[8] C. Pellegrini and J. Plebański, "Tetrad fields and gravitional fields," Matematisk-Fysiske Skrifter udg. af det Kongelige Danske Videnskabernes Selskab, vol. 2, no. 4, 1963.

[9] T. G. Lucas, Y. N. Obukhov, and J. G. Pereira, "Regularizing role of teleparallelism," Physical Review $D$, vol. 80, no. 6, article 064043 .

[10] F. W. Hehl, "Poincare gauge field theory," in Proceedings of the 6th School of Cosmology and Gravitation on Spin, Torsion, Rotation and Supergravity, Erice, Italy, 1979, P. G. Bergmann and V. de Sabbata, Eds., Plenum, New York, NY, USA, 1980.

[11] K. Hayashi, "The gauge theory of the translation group and underlying geometry," Physics Letters B, vol. 69 , no. 4 , pp. 441-444, 1977.

[12] K. Hayashi and T. Shirafuji, “New general relativity,” Physical Review D, vol. 19, no. 12, pp. 3524-3553, 1979.

[13] K. Hayashi and T. Shirafuji, "Addendum to "new general relativity"," Physical Review D, vol. 24, no. 12, pp. 3312-3314, 1981.

[14] M. Blagojević and M. Vasilić, "Asymptotic symmetry and conserved quantities in the Poincare gauge theory of gravity," Classical and Quantum Gravity, vol. 5, no. 9, pp. 1241-1257, 1988.

[15] T. Kawai, "Energy-momentum and angular momentum densities in gauge theories of gravity," Physical Review D, vol. 62, no. 10, article 104014, pp. 1-9, 2000.

[16] T. Kawai, K. Shibata, and I. Tanaka, "Generalized equivalence principle in extended new general relativity," Progress of Theoretical Physics, vol. 104, no. 3, pp. 505-530, 2000.

[17] J. W. Maluf, J. F. DaRocha-Neto, T. M. L. Toribio, and K. H. Castello-Branco, "Energy and angular momentum of the gravitational field in the teleparallel geometry," Physical Review D, vol. 65, no. 12, Article ID 124001, 2002.

[18] R. Arnowitt, S. Deser, and C. W. Misner, "The dynamics of general relativity," in Gravitation: An Introduction to Current Research, L. Witten, Ed., chapter 7, p. 227, Wiley, New York, NY, USA, 1962.

[19] J. W. Maluf, "Hamiltonian formulation of the teleparallel description of general relativity," Journal of Mathematical Physics, vol. 35, no. 1, pp. 335-343, 1994.

[20] J. W. Maluf and S. C. Ulhoa, "On the gravitational angular momentum of rotating sources," General Relativity and Gravitation, vol. 41, no. 6, pp. 1233-1247, 2009.

[21] N. Toma, "A Kerr metric solution in new general relativity," Progress of Theoretical Physics, vol. 86, p. $659,1991$.

[22] M. Ahmed and S. M. Hossain, "Energy localization in curved spacetime," Progress of Theoretical Physics, vol. 93, p. 901, 1995.

[23] G. G. L. Nashed, "Reissner-Nordström solutions and energy in teleparallel theory," Modern Physics Letters A, vol. 21, no. 29, pp. 2241-2250, 2006.

[24] A. A. Sousa, R. B. Pereira, and J. F. da Rocha-Neto, "Angular momentum of the BTZ black hole in the teleparallel geometry," Progress of Theoretical Physics, vol. 114, no. 6, pp. 1179-1190, 2006.

[25] F. Gronwald, "Metric-affine gauge theory of gravity I. Fundamental structure and field equations," International Journal of Modern Physics D, vol. 6, no. 3, pp. 263-303, 1997.

[26] Y. M. Cho, "Einstein Lagrangian as the translational Yang-Mills Lagrangian," Physical Review D, vol. 14, no. 10, pp. 2521-2525, 1976.

[27] U. Muench, U ber teleparallele Gravitationstheorien, Diploma thesis, University of Cologne, 1997.

[28] R. Tresguerres, "Translations and dynamics," International Journal of Geometric Methods in Modern Physics, vol. 5, no. 6, pp. 905-946, 2008.

[29] Yu. N. Obukhov and J. G. Pereira, "Metric-affine approach to teleparallel gravity," Physical Review D, vol. 67, no. 4, article 044016, 2003.

[30] Yu. N. Obukhov and J. G. Pereira, "Lessons of spin and torsion: reply to 'Consistent coupling to Dirac fields in teleparallelism'," Physical Review D, vol. 69, no. 12, article 128502, 2004.

[31] Yu. N. Obukhov, G. F. Rubilar, and J. G. Pereira, "Conserved currents in gravitational models with quasi-invariant Lagrangians: application to teleparallel gravity," Physical Review D, vol. 74, no. 10, article 104007, 2006.

[32] D. Puetzfeld, "An exact-plane fronted wave solution in metric-affine gravity," in Exact Solutions and Scalar Field in Gravity: Recent Developments, A. Macías, J. Cervantes-Cota, and C. Lämmerzahl, Eds., pp. 141-151, Kluwer, Dordrecht, The Netherlands, 2001.

[33] A. García, A. Macías, D. Puetzfeld, and J. Socorro, "Plane-fronted waves in metric-affine gravity," Physical Review D, vol. 62, no. 4, article 044021, pp. 1-7, 2000. 
[34] A. D. King and D. Vassiliev, "Torsion waves in metric-affine field theory," Classical and Quantum Gravity, vol. 18, no. 12, pp. 2317-2329, 2001.

[35] V. Pasic and D. Vassiliev, "Pp-waves with torsion and metric-affine gravity," Classical and Quantum Gravity, vol. 22, no. 19, pp. 3961-3975, 2005.

[36] D. Vassiliev, "Pseudoinstantons in metric-affine field theory," General Relativity and Gravitation, vol. 34, no. 8, pp. 1239-1265, 2002.

[37] D. Vassiliev, “Quadratic metric-affine gravity," Annalen der Physik, vol. 14, no. 4, pp. 231-252, 2005.

[38] Yu. N. Obukhov, "Dirac fermions in strong gravitational fields," Physical Review D, vol. 73, no. 2, article 024025, 2006.

[39] Yu. N. Obukhov, "Poincaré gauge gravity: selected topics," International Journal of Geometric Methods in Modern Physics, vol. 3, no. 1, pp. 95-137, 2006, http://www.worldscinet.com/ijgmmp/.

[40] G. G. L. Nashed, "Energy and momentum of a spherically symmetric dilaton frame as regularized by teleparallel gravity," Annals of Physics, vol. 523, no. 6, p. 450, 2011.

[41] Yu. N. Obukhov and G. F. Rubilar, "Covariance properties and regularization of conserved currents in tetrad gravity," Physical Review D, vol. 73, no. 12, article 124017, 2006. 

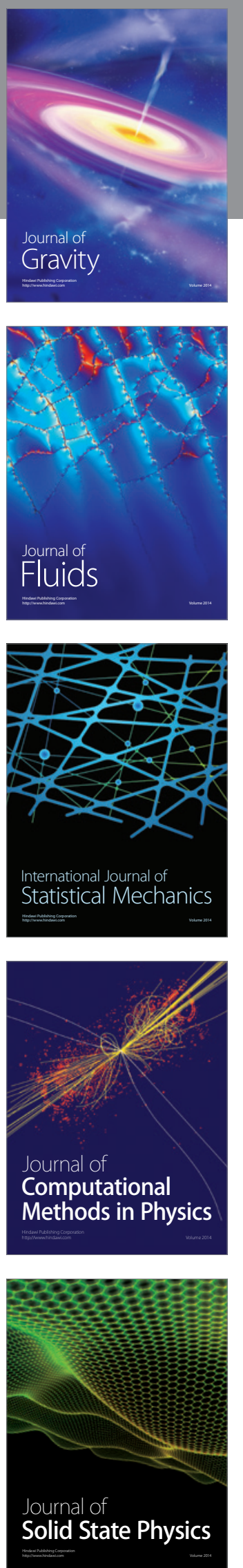

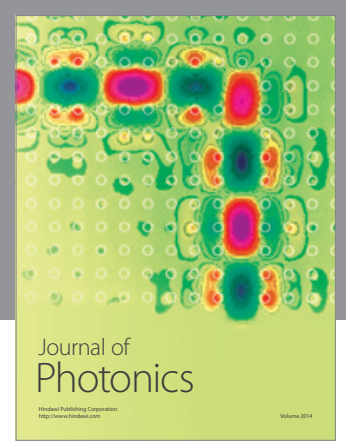

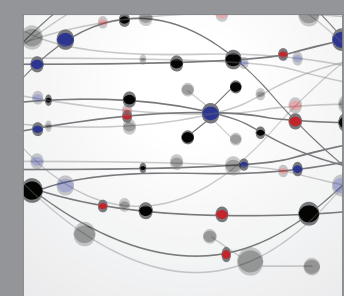

The Scientific World Journal
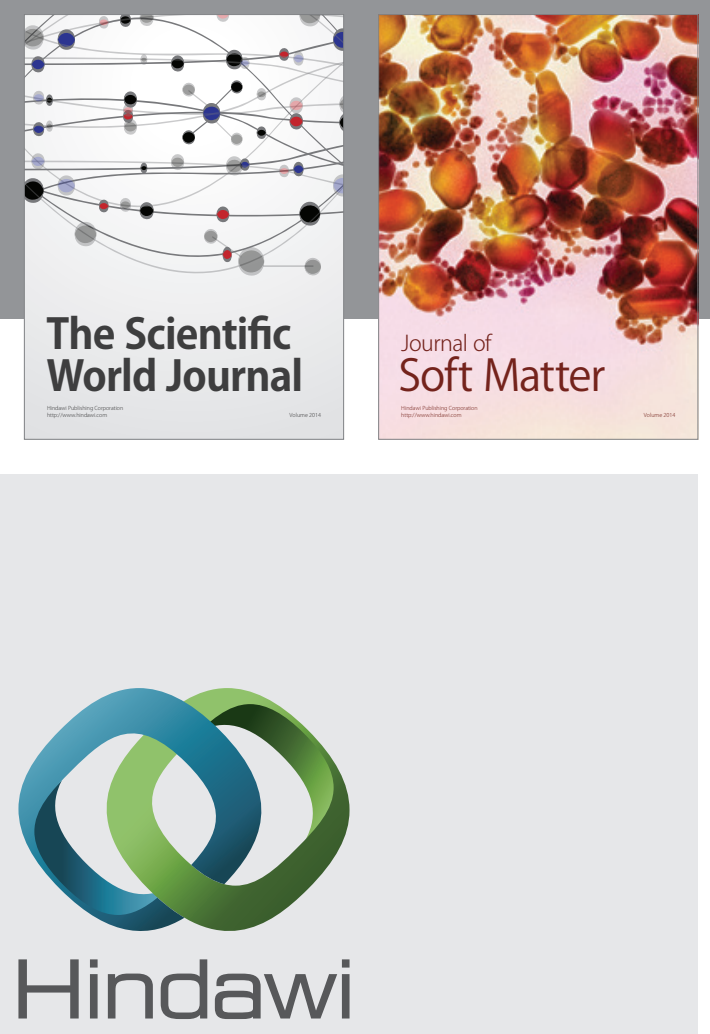

Submit your manuscripts at

http://www.hindawi.com
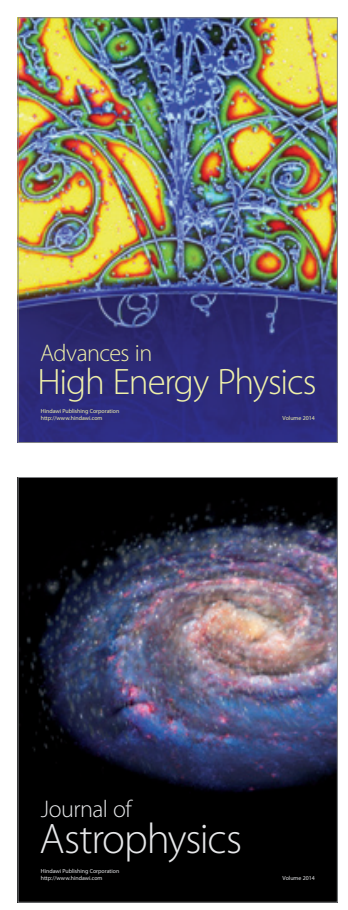
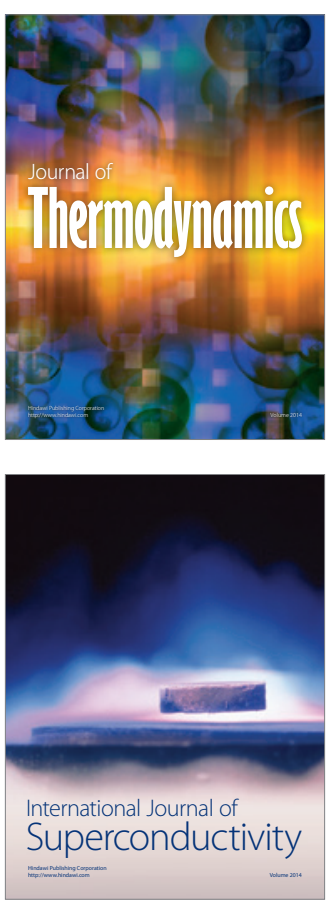
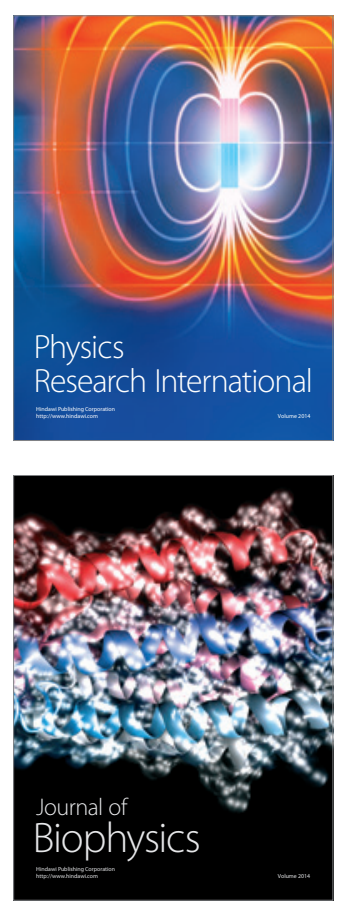
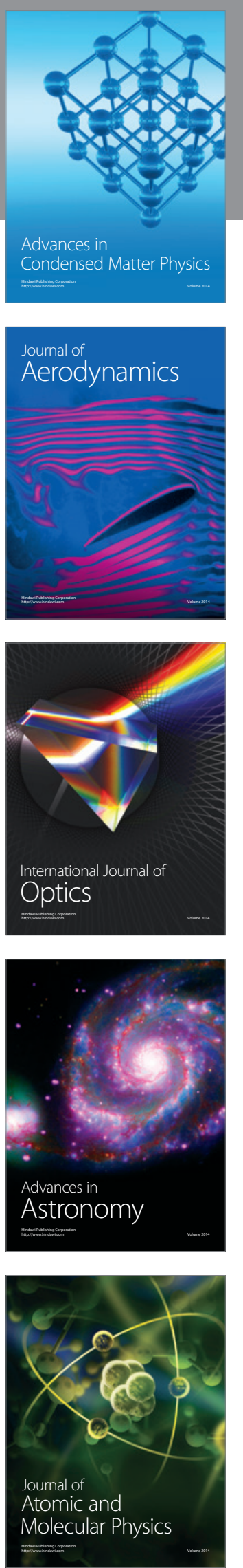